SCIENTIFIC LETTER

\title{
Symptomatic occlusion of the access vein after pacemaker or ICD lead extraction
}

\author{
F A Bracke, A Meijer, L M van Gelder
}

Heart 2003;89:1348-1349

A lthough the North American Society of Pacing and Electrophysiology policy statement on lead extraction does not indicate non-functional leads as either class I or II indications (with the exception of non-functional leads in young patients), there is a tendency to view removal of any non-functional lead as desirable. ${ }^{2}$ The protagonists for this vision argue that leads are neither permanent nor biocompatible, and non-functional leads should therefore pose a risk if not removed. One of the supposed risks is venous occlusion when an increasing number of leads are implanted. However, given the often disruptive nature of freeing the leads from the veins with extraction tools, lead extraction might predispose to thrombosis and venous obstruction. As there has been no follow up study of venous occlusion after lead extraction, we reviewed the incidence of symptomatic occlusions in patients who underwent lead extraction in our institution.

\section{PATIENTS AND METHODS}

Contrast venography of the access vein was available before lead extraction in 89 patients treated in our centre. We interviewed 87 of the 89 patients a mean (SD) of 29 (14) months after lead extraction (two patients died during follow up of non-cardiac causes). There were 70 patients with a pacemaker and 17 with an implantable cardioverterdefibrillator (ICD). The average age was 57 (14) years and 42 were men.

Lead extraction was performed with traction, either directly or with a locking stylet, followed by laser sheath extraction if not successful (Excimer xenon chloride laser, Spectranetics, Colorado Springs, Colorado, USA). Laser application was started from inside the pocket to access the venous circulation. When applicable in patients presenting with lead malfunction, we implanted new leads at the same entry site either after exchanging the laser sheath for a guide wire or after new catheterisation of the subclavian vein. When infection was present, we inserted new leads in the contralateral subpectoral area during follow up if pacing was still considered necessary. Anticoagulation was only started after lead extraction if it was already instituted before the procedure. Clinical follow up was obtained by telephone interview of the patients.

Statistical analysis was performed using a two tailed Fisher's test for contingency tables and Student's $t$ test for numerical observations.

\section{RESULTS}

The results of 65 out of the 87 patients in whom the entry vein was patent before extraction are summarised in table 1 . Oedema as a symptom of venous occlusion occurred in five patients. In two patients it resolved spontaneously after several months, but in one patient it gave rise to Sudeck's atrophy. All symptoms occurred after the use of a laser sheath, but the difference with traction was not significant $(\mathrm{p}=0.57)$. Of note, the time from implant of the oldest removed lead with laser was significantly longer than with traction (82 (56) months $v 34$ (47) months, respectively; $\mathrm{p}=0.005)$ and the number of leads extracted per patient was higher when a laser sheath was used (2.1 (0.7) $v 1.7$ (0.5); $\mathrm{p}=0.05)$.

Neither the indication for extraction nor the type of lead extracted (pacemaker or ICD) were related to the occurrence of new symptoms. All but one of the 28 patients with superfluous non-functional leads had new leads inserted ipsilaterally during the same procedure.

In the 22 patients with known occlusion before lead extraction two were symptomatic before the procedure. Eighteen of them presented with infection, and a laser sheath was necessary in 21 patients. Two previously asymptomatic patients, both presenting with infection, had cyanosis but not oedema of the arm after extraction.

\section{DISCUSSION}

We found an $8 \%$ incidence of new symptoms suggestive of venous occlusion following lead extraction in patients with a previously patent entry vein. Given the underestimation of angiographic venous obstruction by clinical symptoms, the incidence of new angiographic obstruction after lead extraction could still be higher. ${ }^{3}$

Table 1 Incidence of symptomatic venous occlusion in patients with a patent access vein before extraction

\begin{tabular}{|c|c|c|c|c|c|}
\hline & & \multirow[b]{2}{*}{$\mathbf{n}$} & \multicolumn{2}{|c|}{ Symptomatic } & \multirow[b]{2}{*}{ p Value } \\
\hline & & & $\mathbf{N}$ & $\%$ & \\
\hline \multirow{2}{*}{ Extraction technique } & Traction & 13 & 0 & 0 & \multirow{2}{*}{0.58} \\
\hline & Laser & 52 & 5 & 10 & \\
\hline \multirow{2}{*}{ Indication } & Non-functional & 28 & 4 & 14 & \multirow{2}{*}{0.15} \\
\hline & Infection & 37 & 1 & 3 & \\
\hline \multirow{2}{*}{ PM/ICD } & $I C D$ & 14 & 2 & 14 & \multirow{2}{*}{0.29} \\
\hline & PM & 51 & 3 & 6 & \\
\hline Total & & 65 & 5 & 8 & - \\
\hline
\end{tabular}

$I C D$, implantable cardioverter-defibrillator; PM, pacemaker. 
All new cases occurred after the use of a laser sheath but the difference with traction did not reach significance. Further, there were important differences between both patient groups: the time from implant was significantly longer and the number of leads extracted per patient was higher when the laser sheath was used. Finally, as no other superior extraction techniques were used it cannot be concluded that venous occlusion was specific for laser extraction.

Infection could be a confounding factor with regard to venous occlusion. However, our study population included $63 \%$ of patients with infected pacing or ICD systems, but the incidence of new symptoms did not differ significantly between patients presenting with infection or with nonfunctional leads.

The insertion of new leads might have influenced occlusion after lead extraction for non-functional leads, as all but one patient had new leads inserted during the extraction procedure at the same entry site. But in a prospective study by de Cock and colleagues there were no differences with respect to thrombotic complications in 48 patients with multiple leads (39 patients with three leads, nine patients with four leads) versus 48 age matched control patients with dual chamber pacemakers. ${ }^{4}$ Additionally, we showed from pre-extraction angiograms that there is no relation between the number of leads and venous occlusion, thus making the insertion of new leads less likely to cause obstruction. ${ }^{5}$

In conclusion, rather than be protective, our study indicates that lead extraction itself might cause venous obstruction. Although these data should be confirmed by angiographic studies, until then it is prudent to refrain from lead extraction for the sole purpose of preventing venous occlusion.

\section{Authors' affiliations \\ F A Bracke, A Meijer, L M van Gelder, Department of Cardiology, Catharina Hospital, Eindhoven, The Netherlands}

Correspondence to: Dr Frank A Bracke, Department of Cardiology, Catharina Hospital, 2 Michelangelolaan, PO Box 1350, 5602 ZA Eindhoven, The Netherlands; f.bracke@skynet.be

Accepted 19 June 2003

\section{REFERENCES}

1 Love CJ, Wilkoff BL, Byrd CL, et al. Recommendations for extraction of chronically implanted transvenous pacing and defibrillator leads: indications, facilities, training. North American Society of Pacing and Electrophysiology lead extraction conference faculty. Pacing Clin Electrophysiol 2000;23:544-51.

2 Byrd CL. Advances in device lead extraction. Curr Cardiol Rep 2001;3:324.

3 Bracke FA, Meijer A, van Gelder LM. Pacemaker lead complications: when is extraction appropriate and what can we learn from published data? Heart 2001;85:254-9.

4 De Cock CC, Vinkers M, Van Campe LC, et al. Long-term outcome of patients with multiple ( $>$ or $=3$ ) noninfected transvenous leads: a clinical and echocardiographic study. Pacing Clin Electrophysiol 2000;23:423-6.

5 Bracke FA van Gelder LM, Meijer A. Venous occlusion of the access vein in patients referred for lead extraction: influence of patient and lead characteristics. Pacing Clin Electrophysiol 2003;26:1649-52.

\section{FROM BMJ JOURNALS}

\section{Detection of early impairment of coronary flow reserve in patients with systemic sclerosis}

R Montisci, A Vacca, P Garau, P Colonna, M Ruscazio, G Passiu, S lliceto, A Mathieu

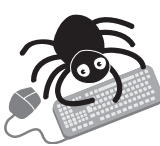

Please visit the Heart website [www.heartjinl. com] for a link to the full text of this article.
Objective: To investigate whether coronary flow reserve (CFR), measured by a new noninvasive method, is impaired early in patients with systemic sclerosis (SSc) and whether CFR impairment correlates with clinical or functional measures, or both.

Methods: 27 patients with SSc without clinical evidence of ischaemic heart disease and 23 control group subjects matched for age and sex were studied. CFR was evaluated in the left anterior descending coronary artery (LAD) with a new non-invasive method: contrast (Levovist) enhanced transthoracic Doppler during adenosine infusion. The pulsed wave Doppler examination of blood flow velocity was recorded in the LAD at rest and after maximum vasodilatation by adenosine infusion.

Results: In patients with SSc, without clinical evidence of ischaemic heart disease, CFR was impaired $(p=0.0001) .14 / 27$ patients with SSc had severe reduction of the CFR $(\leqslant 2.5)$ compared with controls $(p=0.002)$. A non-significant trend between mean CFR and the severity and duration of the disease was also seen.

Conclusions: CFR is often reduced in patients with SSc, suggesting early preclinical cardiac involvement in SSc. This impairment in coronary microvasculature is detectable by a noninvasive echocardiographic method and in this study was more common in the diffuse form of SSc.

A Annals of the Rheumatic Diseases 2003;62:890-893. 